\title{
Serum Antibody Titers of Measles and Rubella among Japanese Children
}

\author{
Yuichirou Tsuji*, Chitose Karasawa \\ Department of Pediatrics, Ikegami General Hospital, Tokyo, Japan \\ Email: "yuitsuji@ybb.ne.jp
}

Received 27 September 2015; accepted 27 October 2015; published 30 October 2015

Copyright (C) 2015 by authors and Scientific Research Publishing Inc.

This work is licensed under the Creative Commons Attribution International License (CC BY). http://creativecommons.org/licenses/by/4.0/

(c) (i) Open Access

\begin{abstract}
Background: The rubella outbreak in Japan has not yet been eliminated. In particular, an outbreak of congenital rubella syndrome has recently become a public health problem in Japan. The World Health Organization has set an elimination target of 2015 for measles in Japan. However, an outbreak of measles occurred in Japan between 2007 and 2008. Starting in April 2006, the measles and rubella vaccines were administered twice, once when a child reached 1 year old and once when the child was 5 - 6 years old (just before starting elementary school). Between October of 1978 and 2006, children were vaccinated only once for measles and rubella. Design: During the study enrollment period (2011-2013), the serum antibody titers of measles and rubella were measured in pediatric patients $(n=163)$ in the Tokyo Takanawa Hospital. Results: The prevalence rates of the two diseases indicated that only one vaccination was insufficient to protect against infection. Conclusions: Our studies have determined that it was important to vaccinate children twice for measles and rubella during early infancy.
\end{abstract}

\section{Keywords}

Measles, Rubella, Immunization, Prevalence Rate, Japanese Children

\section{Introduction}

Rubella is still prevalent periodically in Japan. It was most prevalent between 2007 and 2008. In 2008, there were 11,005 cases of measles in Japan. In 2014, there were 463 cases. In 2015, the World Health Organization (WHO) announced efforts to eliminate measles in Japan.

Rubella was prevalent in 2013. During this time, there were 14,344 rubella cases in Japan. The cases were almost always domestic. Most of the patients were adult males, because males did not receive the rubella vac-

*Corresponding author. 
cine through the national vaccination program until April of 1979. As a consequence, there were 32 patients who were born with congenital rubella syndrome (CRS) in 2013 out of a total of 1,029,800 live births.

We think that the reason for the increased prevalence was that the two vaccinations were only inoculated once until recently.

The measles and rubella vaccines were added to the childhood vaccination program in Japan in 1976. Rubella vaccination began in 1977 among junior high school females (a single dose) and measles vaccination started in 1978 for male and female children, but the vaccines were also only administered once.

In 2006, a routine two-dose combined measles and rubella (MR) live attenuated virus vaccination schedule was initiated in Japan for 1 and 5 - 6 years old children. Therefore, we examined the antibody titers of measles and rubella in serum from pediatric patients in our hospital. This study aimed to determine the importance of a two-dose universal immunization program with the measles and rubella vaccines.

\section{Methods}

The study was conducted between April-2011 and September-2013.

\subsection{Measurements Made Prior to the Examinations}

The measurements of titers were performed only when a blood test was medically indicated. We did not performed blood tests solely for the purpose of antibody measurement. We checked the date of measles and rubella vaccination on maternal children handbook. Written informed consent in Japanese obtained from guardian of all patients.

\subsection{Methods for Antibody Measurement}

All laboratory testing was blinded without knowledge of the vaccination statuses of the individuals.

\subsubsection{Measles: The Titer of Immunoglobulin G (IgG) Evaluated by Enzyme Immunoassay (EIA)}

The measurements were performed using an automated, multi-parametric immunoassay testing system (VIDAS ${ }^{\circledR}$, bioMerieux, France) in our central examination center. The negative standard value was $<0.5$ according to the Central Celinical Laboratory of Shimane University Hospital.

\subsubsection{Rubella: Evaluated Using Hemagglutination Inhibition (HI) Tests in the SRL (Japan)} Seronegativity was based on the manufacturer's threshold of $<8$ rubella international units (RIUs).

\subsection{Participants}

A total of 163 children (88 males and 75 females) who were 1 - 15 years of age were enrolled in the study. The average age was $7.34 \pm 3.01$ years $( \pm 1$ standard deviation). We excluded children who had not received the measles or rubella vaccines and who had no history of the disease. However, we did not consider whether there was a history of contact of these individuals with patients who had either disease. The age-specific numbers of participants are shown in Figure 1.

We were using Microsoft ${ }^{\circledR}$ Excel 2010 on the results of the statistical process.

\section{Results}

There were no children who had a history of the natural incidence on both diseases.

The number of participants who were not vaccinated or who were vaccinated once or twice are shown in Figure 2.

The distribution of HI antibody to rubella $(\mathrm{n}=157)$ is shown in Figure 3.

The distribution of IgG antibody to measles $(\mathrm{n}=163)$ is shown in Figure 4.

The negative rate of titer is shown in Figure 5.

The seronegative rate of rubella was $7.0 \%$ in total. It was $11.8 \%$ with single vaccination, but dropped to $3.4 \%$ with double vaccination.

The seronegative rate of measles was $11.0 \%$ in total. It was $13.5 \%$ with single vaccination, but dropped to $9.0 \%$ with double vaccination. 


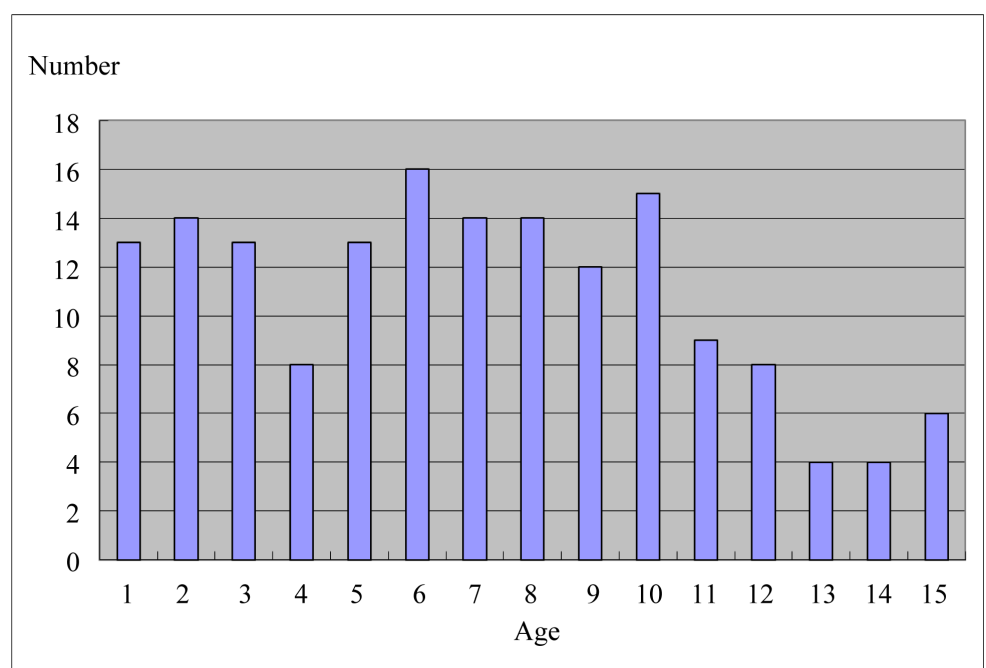

Figure 1. Age-specific numbers $(\mathrm{n}=163)$.

\begin{tabular}{cccc}
\hline Vaccine & Patient & Non-vaccinated & Vaccinated \\
\hline Rubella & 0 & $6(3.7 \%)$ & Once: 68 \\
& 0 & $0(0 \%)$ & Twice: 89 \\
Measles & & & Once: 74 \\
& & & Twice: 89
\end{tabular}

Figure 2. Number of patients who were not vaccinated, vaccinated once, or vaccinated twice.

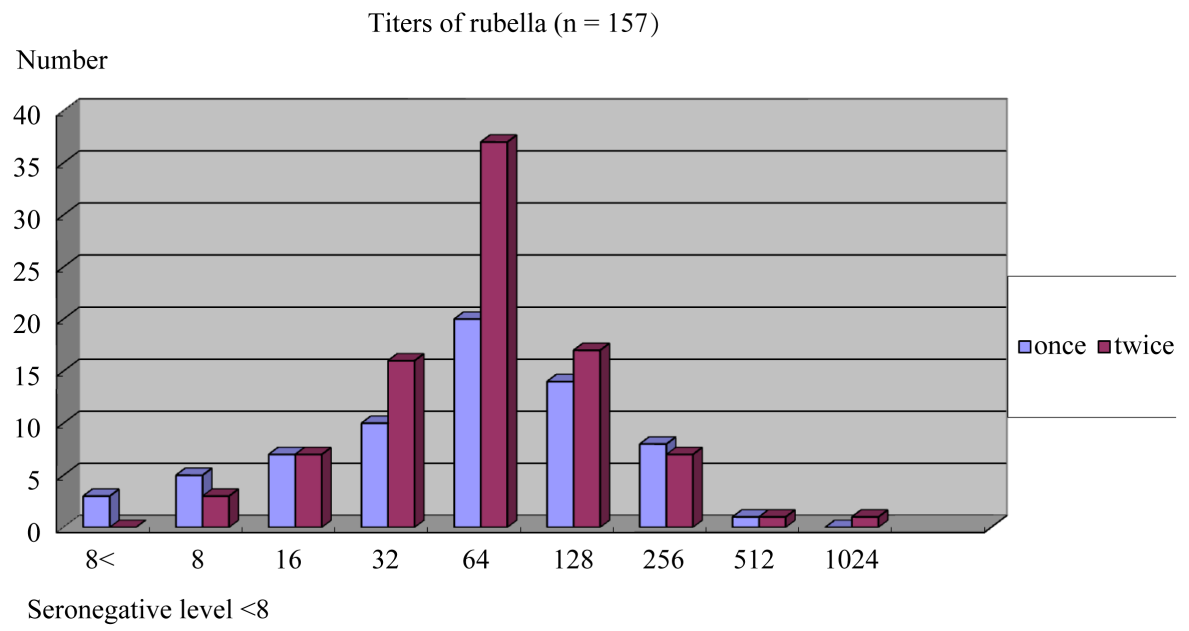

Figure 3. Hemagglutination inhibition levels.

\section{Discussion}

The rate of negative or equivocal antibody levels to rubella was $11.8 \%$ after a single dose of the vaccine and the rate of negative or equivocal antibody levels to measles was $13.5 \%$ after a single dose. Therefore, a single dose was not sufficient to achieve the required antibody levels. In the group of individuals who received a second 


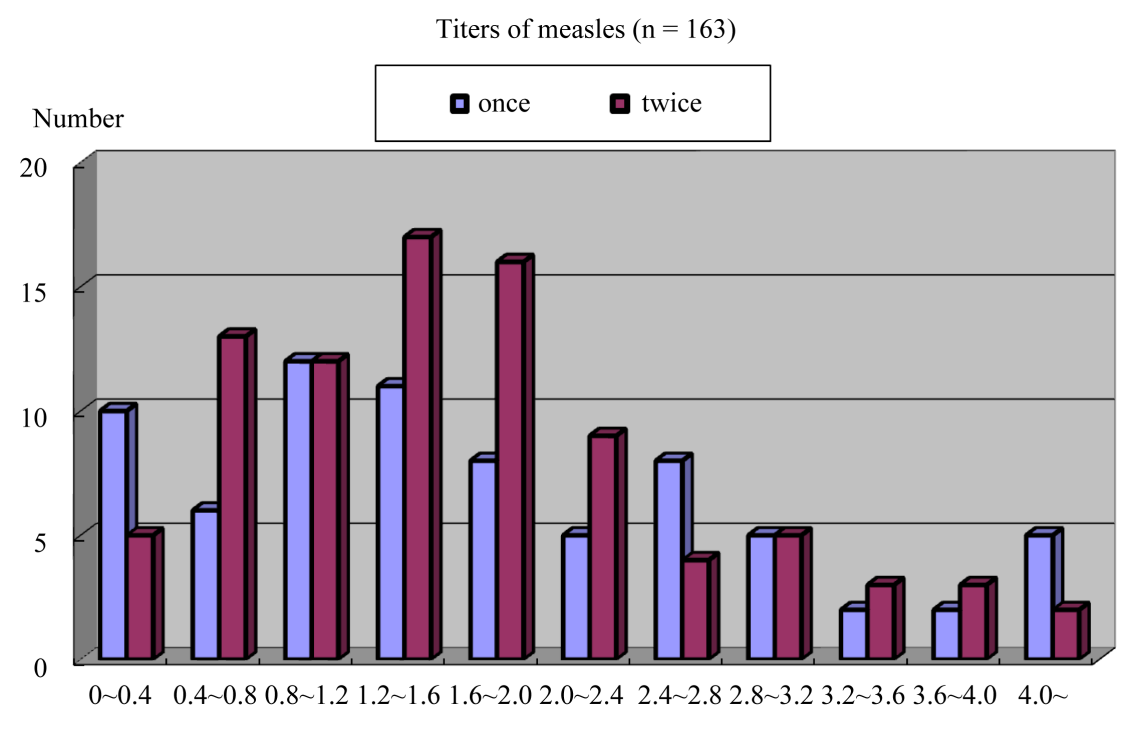

Seronegative level $<0.50$

Figure 4. Enzyme immunoassay levels.

\begin{tabular}{rc}
\hline Vaccine & Rate \\
\hline Rubella (total) & $7.0 \%(11 / 157)$ \\
(once) & $11.8 \%(8 / 68)$ \\
(twice) & $3.4 \%(3 / 89)$ \\
Measles (total) & $11.0 \%(18 / 163)$ \\
(once) & $13.5 \%(10 / 74)$ \\
\hline
\end{tabular}

\section{Figure 5. Seronegative rates.}

dose, the rates of seronegativity for rubella and measles decreased to $3.4 \%$ and $9.0 \%$ respectively. It was not possible to determine whether this resulted from primary or secondary vaccine failure in this study. The Centers for Disease Control schedule for routine childhood immunization states that the second dose should routinely be administered prior to the start of school ( 4 - 6 years of age). Currently the MR vaccine is administered according to a similar schedule in Japan. The time between the first and the second vaccinations is approximately 4 - 5 years, and it is expected that primary vaccine failure exists in children based on the ratio of uniformity in our study. It is thought that two doses of the rubella vaccine are necessary to prevent an outbreak and the development of CRS. The main cause of the recent outbreak of rubella in Japan was adults who had received only a single dose or no vaccine in the past.

The underlying cause of seronegative levels in a patient who was vaccinated twice for rubella and measles is unclear. In Japan, it is currently difficult to consider the possibility of failure and/or the incorrect delivery of vaccines. Tan et al. reported that low antibody levels may be caused by individual immunogenetic factors [1].

The vaccination rate for the MR vaccine was $95.5 \%$ at the first dose and $93.0 \%$ at the second dose in Japan in 2013 [2]. It is thought that rates $>95 \%$ are necessary to control the outbreak. The second vaccination rate was lower. It is therefore necessary to improve the vaccination rate in Japan.

In our clinical study, age, gender and the period after vaccination did not affect the results. Furthermore, it did 
not consider a past history of contact with individuals with subclinical infections. However, there has been little opportunity for natural boosting in Japan currently. In our study, there was no effect on measles and/or rubella. Thus, it is thought that the results of this report are representative of the current trends among Japanese children.

On the other hand, the relationship between clinical protection and levels for the diseases is not clear. The protective threshold for enzyme-linked immunosorbent assay-based antibody levels of measles and the HI titer of rubella has not been established. However, it can be estimated based on antibody levels in clinical practice. Chen et al. reported that specific antibody concentrations are protective against measles [3]. In contrast, there were also reports that low antibody levels among vaccinated individuals were not indicative of a lack of clinical protection [4] [5]. Pebody et al. suggested that in highly vaccinated populations, the total measles and rubella IgG antibody levels may not accurately reflect protection [6]. Tabatabaei et al. presented that antibody levels were below the assay cut-off levels against measles and rubella in approximately one-fourth of the children following primary MR vaccination [7]. Charles et al. reported that the titers of rubella after two doses of the measles, mumps, and rubella vaccine decreased to pre-second administration levels after 12 years [8].

Two problems still exist. The first is that the domestic EIA value of measles has not yet been standardized to an international unit, and the second is that there is no rule for the negative standard value of rubella HI. The standard threshold in the United States is $<10$ RIU, and the common European threshold is $<15$ RIU. However, the threshold is still controversial in Japan. Therefore, a standard value has not yet been determined in Japan. Thus, the rate of seronegativity is increased and decreased by the standard threshold. These issues will require additional examination in the future.

In summary, a single vaccination is not sufficient to prevent infection with rubella and measles. A second dose of the vaccines is important and necessary in order to prevent infections and outbreaks. Paunio et al. reported that individuals who were vaccinated twice were better protected against measles than were individuals who only received a single dose of the measles vaccine [9]. Additional studies are required to determine the appropriate time period between administration of the first and second doses, and to determine whether a third dose is necessary.

In conclusion, two doses of the vaccines for rubella and measles is important for prevention of rubella and measles. Additional improvement of the vaccination rate is necessary. Accurate and continuous monitoring will also be necessary for continuation of elimination in measles in Japan. Furthermore, it is necessary to vaccinate adults who have no history of the measles and rubella vaccines in order to prevent outbreaks and especially to eliminate cases of CRS.

\section{Conclusion}

It is important to vaccinate two doses of rubella and measles for prevention of rubella and measles and to keep the elimination of measles in Japan. Furthermore, prevention of outbreaks of rubella is a pressing problem now in Japan.

\section{Consent}

The personal information that could be used to identify individuals was not included in this article.

\section{Ethical Approval}

This study was performed with the permission of the Tokyo Takanawa Hospital Ethics Committee including outside members who were teachers and lawyers. All the authors hereby declare that all experiments were examined and approved by the ethical standards stated in the 1964 Declaration of Helsinki.

\section{Financial Disclosure}

This study was conducted using research funds from Tokyo Takanawa Hospital. No funding was received from the manufacturers of the vaccine.

\section{Conflict of Interest Statement}

The authors declare that there are no conflicts of interest. 


\section{References}

[1] Tan, P., Jacobson, R.M., Poland, G.A., et al. (2001) Twin Studies of Immunogenicity-Determining the Genetic Contribution to Vaccine Failure. Vaccine, 19, 2434-2439.

[2] www.mhlw.go.jp/bunya/keuko/kekkaku-kansenshou21/hashika.hytml

[3] Chen, R.T., Markowitz, L.E., Albrecht, P., et al. (1990) Measles Antibody: Re-Evaluation of Protective Titers. Journal of Infectious Diseases, 162, 1036-1042. http://dx.doi.org/10.1093/infdis/162.5.1036

[4] Vitek, C.R., Aduddel, M., Brinton, M.J., et al. (1999) Increased Protections during a Measles Outbreak of Children Previously Vaccinated with a Second Dose of Measles-Mumps-Rubella Vaccine. The Pediatric Infectious Disease Journal, 18, 620-623. http://dx.doi.org/10.1097/00006454-199907000-00010

[5] Hennessey, K.A., Ion-Nedelcu, N., Craciun, M., et al. (1999) Measles Epidemic in Romania, 1996-1998: Assessment of Vaccine Effectiveness by Case-Control and Cohort Studies. American Journal of Epidemiology, 150, 1250-1257. http://dx.doi.org/10.1093/oxfordjournals.aje.a009952

[6] Pebody, R.G., Gay, N.J., Hesketh, L.M., et al. (2002) Immunogenicity of Second Dose Measles-Mumps-Rubella (MMR) Vaccine and Implications for Serosurveillance. Vaccine, 20, 1134-1140. http://dx.doi.org/10.1016/S0264-410X(01)00435-2

[7] Tabatabaei, S.R., Esteghamati, A.R., Shiva, F., Fallah, F., Radmanesh, R., Abdinia, B., Shamshiri, A.R., Khairkhaha, M., Ebrahimabad, H.S. and Karimi, A. (2013) Detection of Serum Antibodies against Measles, Mumps and Rubella after Primary Measles, Mumps and Rubella (MMR) Vaccination in Children. Archive of Iranian Medicine, 16, 38-41.

[8] LeBaron, C.W., Forghani, B., Matter, L., Reef, S.E., Beck, C., Bi, D., Cossen, C. and Sullivan, B.J. (2009) Persistence of Rubella Antibodies after 2 Doses of Measles-Mumps-Rubella Vaccine. Journal of Infectious Diseases, 200, 888-899. http://dx.doi.org/10.1086/605410

[9] Paunio, M., Peltona, H., Valle, M., et al. (1999) Twice Vaccinated Recipients Are Better Protected against Epidemic Measles than Are Single Dose Recipients of Measles Containing Vaccine. Journal of Epidemiology and Community Health, 63, 173-178. http://dx.doi.org/10.1136/jech.53.3.173 